\title{
Reflexiones acerca de una experiencia de "gamificación" en Derecho mercantil dentro del grado de ADE de la Universitat de Barcelona
}

Reflections about an experience of "gamification" in business law on the $A D E$ degree of the Universitat of Barcelona

\author{
Noemí Jiménez Cardona \\ Profesora-Investigadora de Derecho Mercantil \\ Miembro integrante del Grupo de Innovación Docente Dikasteia \\ Departamento de Derecho Privado \\ Universitat de Barcelona, España \\ E-mail: njimenezcardona@ub.edu
}

\begin{abstract}
Resumen: El presente artículo centra su atención en la experiencia docente (innovación) que, como profesora e investigadora de la Sección de Derecho Mercantil del Departamento de Derecho Privado de la Universitat de Barcelona, he desarrollado en la disciplina de Derecho Mercantil del Grado de ADE, impartido en la Facultad de Economía y Empresa, a lo largo del curso académico 2018-2019. Dicha experiencia tiene que ver con la utilización puntual, en cuanto complemento de las clases teóricoexpositivas, de diferentes técnicas de "gamificación”. La aplicación de estos recursos a través de la introducción de las dinámicas y componentes de los juegos, junto con el trabajo en equipo, ha resultado especialmente útil en orden a corregir la tradicional desmotivación de parte del estudiantado en el aula, su falta de asistencia a clase, o la ausencia de implicación real de los estudiantes universitarios en un proceso de aprendizaje en el que no solo el papel protagonista debe ser compartido entre el profesor y el estudiantado, sino en el que también resulta obligado afrontar su formación tanto en contenidos como en competencias y habilidades profesionales.
\end{abstract}

Palabras clave: metodologías docentes activas, gamificación, ludificación, aprendizaje, conocimientos, competencias y habilidades. 
Abstract: This article focuses on the high educational experience (innovation) that, as researcher of the Business Law Section of the Department of Private Law of the University of Barcelona, I have developed in the Business Law discipline of the ADE Degree, taught in the Faculty of Economics and Business, throughout the academic year 2018-2019. This experience is related to the timely use, as a complement to the master classes, of different gamification skills. The application of these resources through the introduction of the dynamics and components of the games, along with the teamwork, they are particularly relevant in order to correct the traditional demotivation of students in the classroom, their lack of class attendance, or the lack of real commitment of university students in a learning process in which not only the leading role must be shared between the teacher and the student body, but in which it is also forced to face their training both in knowledge and vocational skills and competences.

Keywords: active learning methodologies, gamification, playful activities, learning, knowledge, skills and competences.

\section{Necesidades docentes cambiantes en el aula universitaria. Las metodologías docentes activas}

La docencia, junto con la investigación y la transferencia de conocimiento y tecnología (innovación), es uno de los ejes principales y señas de identidad de la actividad universitaria (BENITO et al., 2018, 153-175). Lo que una Universidad ha sido, es y seguirá siendo viene determinado, en buena medida, por qué enseña y cómo lo enseña, de tal forma que adquiere notable relevancia el llamado proceso de transmisión de conocimiento, integrado por dos componentes: el primero, estratégico y proactivo, dirigido a dar respuesta efectiva a las necesidades y demandas de la sociedad y el tejido productivo (MARTÍNEZ, 2006, 85-87); y el segundo, operativo, que pone su acento en hacer todo lo posible para alcanzar el cumplimiento efectivo del servicio público de educación superior en cuanto a la formación de las sucesivas generaciones de estudiantes que acceden a la Universidad (BUXARRAIS y BURGUET, 2013, 90-92).

Este componente operativo, como es lógico, no puede ser ajeno a la toma en consideración de las necesidades docentes, ciertamente cambiantes, que se manifiestan a diario en las aulas universitarias. Como indica PÉREZ CABRERA (2013, 45-62) las nuevas generaciones de estudiantes, ni mejores ni peores que sus predecesoras, pero sí 
bien distintas, responden, quizás con una más limitada cultura del sacrificio y el esfuerzo, a muy variados perfiles sociodemográficos, así como disponen de habilidades e instrumentos de comunicación impensables hasta hace muy pocos años (vgr. alumnos digitales y más amplia formación idiomática).

Todo ello hace necesario replantearse, en especial en el campo de las ciencias jurídicas, el sistema tradicional de aprendizaje y evaluación de los estudiantes universitarios, caracterizado por situar su centro de gravedad en el conocimiento de la materia (contenidos) y la figura activa del profesor en el aula (ANDRÉS, 2012, 3-5). De hecho, el proceso de reflexión en torno a un modelo de aprendizaje en el que, de una parte, el papel protagonista sea compartido por el profesor y el estudiantado; y, de otra, también se preste atención, mediante la potenciación de diferentes líneas de innovación docente, a la asunción de competencias y habilidades profesionales de quienes están llamados a convertirse en los profesionales jurídicos del futuro; ha alcanzado su máxima representación desde la entrada en vigor en 2010 del Plan Bolonia (VALLESPÍN, 2013, $8-15)$

Convertir la enseñanza en un "acto compartido" a través de la utilización de nuevas metodologías docentes, plenamente compatibles con las tradicionales, constituye uno de los retos actuales y de futuro del profesorado universitario (PÉREZ CABRERA, 2013, 52), máxime ante la implantación del crédito europeo (FONT, 2014, 36-41). Siendo esto así, en los últimos años se ha convertido en habitual hacer mención del aprendizaje en entornos virtuales, el aprendizaje profesionalizador, la evaluación continuada, la formación de equipos docentes y la incorporación en el aula universitaria de las denominadas metodologías docentes activas (aprendizaje autónomo ${ }^{1}$, aprendizaje colaborativo $^{2}$, aprendizaje entre iguales ${ }^{3}$, aprendizaje servicio ${ }^{4}$, aprendizaje basado en problemas $-\mathrm{ABP}^{5}$, simulaciones y casos prácticos 6 , aula inversa ${ }^{7}$ y “gamificación”).

\footnotetext{
1 Orientado a la mejora de los mecanismos de aprendizaje autónomo del estudiantado, con especial referencia a la vinculación de su aprendizaje con el trabajo en el aula y el trabajo colaborativo o en equipo (vid. CÁRCEL CARRASCO, F. J. (2016). "Desarrollo de habilidades mediante el aprendizaje autónomo", 3c Empresa: investigación y pensamiento crítico, 5 (3), 52-60).

${ }^{2}$ Basado, de una parte, en la distribución de roles, responsabilidades y tareas por lo que hace referencia al desarrollo de actividades que requieren la cooperación y colaboración entre alumnos; y, de otra, en la toma en consideración de instrumentos de evaluación tanto del resultado final como del proceso desarrollado y el nivel de implicación de cada uno de los participantes (vid. DOMINGO, J. (2008). "El aprendizaje cooperativo", Cuadernos de trabajo social, 21, 231-246).

3 Metodología que pretende aprovechar la diversidad en los procesos de enseñanza, aprendizaje y construcción del conocimiento, mediante la fijación de mecanismos de evaluación, control y autogestión del trabajo realizado por cada uno de los implicados (vid. ESCUDERO ESCORCA, T., RODRÍGUEZ, L.
} 


\section{La "gamificación" en el aula universitaria (planteamiento general)}

La idea de introducir estructuras de juego en el marco de actividades que pueden resultar anodinas no es nueva, pues desde siempre ha tenido cabida en contextos como el educativo o empresarial. Sin embargo, en los últimos años, primero en la educación infantil y primaria, y más recientemente, por lo que hace referencia al aula universitaria, la apuesta por los elementos del juego ha adquirido una notable difusión en cuanto metodología docente activa, toda vez que con ella se promueve la motivación por el aprendizaje, se permite la retroalimentación constante, se facilita un aprendizaje atractivo, se fideliza al estudiante y se le otorga un sentimiento de compromiso para con su formación, y se combina la competitividad con el espíritu del trabajo en equipo y la colaboración (ESTEVE, GISBERT, 2011, 55-69).

Concretamente, la exposición se centrará en la experiencia docente adquirida en el Grado de ADE durante el curso académico 2018-2019 en orden a la utilización de la "gamificación" o "ludificación". La "gamificación" puede definirse como una técnica específica de aprendizaje activo que se encuadra dentro de la corriente educativa de la "ludificación", y cuyo propósito principal no es otro que trasladar la mecánica y dinámica del juego al ámbito educativo-profesional con el objetivo de hacer posible que el alumno desarrolle su aprendizaje de forma más atractiva y estimulante. De acuerdo

M. y FERNÁNDEZ, R. (2002). "Aprendizaje entre iguales y construcción de conceptos”, Infancia y Aprendizaje: Journal for the Study of Education and Development, 25 (3), 279-285).

${ }^{4}$ El aprendizaje servicio (APS) implica la integración de procesos de aprendizaje y servicio a la comunidad en proyectos educativos con vinculación y utilidad social, de tal forma que el aprendizaje adquiere un sentido de transferencia e implicación en el propio entorno social (vid. NAVAL, C. et al. (2011). "Ethical and civic education and the social commitment of university students", Encounters on Education, 12, 77-91).

${ }^{5} \mathrm{El} \mathrm{ABP}$, en cuanto estrategia educativa a no confundir con el método del caso práctico, es un método de aprendizaje que permite a los alumnos construir sus conocimientos mediante el planteamiento y resolución de diferentes escenarios-problemas. Es el propio alumno quien debe movilizarse en la búsqueda de recursos, pues a él le corresponde formar su propio conocimiento, por ejemplo, en el caso de las ciencias jurídicas, mediante la búsqueda de las fuentes legales, la recopilación de las doctrinales, y el análisis de la jurisprudencia. Y todo ello con el objetivo de que, más tarde, sea capaz de formular las posibles hipótesis de solución del caso y argumentar su posicionamiento (vid. FONT RIBAS, A. (2012). "El aprendizaje basado en problemas en la formación judicial", Revista de educación y derecho Education and Law Review, 6, 4-8).

${ }^{6}$ Mecanismos que persiguen mejorar la implicación de los alumnos, situándolos ante un contexto real, en cuanto a la resolución de diferentes situaciones y la toma de decisiones. Sobre la aplicación del método del caso práctico en el terreno de las ciencias sociales, en especial las jurídicas, véase, por todos: VALLESPÍN PÉREZ, D. (2011). "El método del caso como instrumento de aprendizaje en el Derecho Procesal”, Revista Educación y Derecho - Education and Law Review, 3, 1-10.

${ }^{7}$ La metodología del aula inversa (flipped classroom) supone un enfoque de la enseñanza universitaria en la que se invierte la tradicional secuencia de actividades formativas: enseñanza-estudio-evaluación, por una nueva en la que se parte del estudio, se procede a la evaluación y se culmina con la enseñanza. Sobre el aula inversa, por todos, véase ESTEVE, A. (2016). "Flipped teaching o la clase invertida en la enseñanza del Derecho", Actualidad Jurídica Iberoamericana, 4 extra, 75-95. 
con KAPP (2012, 198-201) el objetivo último de la "gamificación" consiste en persuadir al estudiante universitario mediante la conversión de una simple tarea, en ocasiones algo monótona, por un reto atractivo en el que éste se involucre de forma activa.

Este fenómeno educativo, si bien es cierto que ha adquirido en los últimos tiempos una cierta popularidad, no lo es menos que ha existido, aun cuando inconscientemente, desde siempre. Así lo expresa PRENSKY (2000, 20-22) cuando, por ejemplo, como profesores premiamos a nuestros estudiantes con los clásicos "puntos positivos", en realidad existe cierta "gamificación", ya que todo trabajo bien realizado se acompaña de la oportuna "recompensa". Otra cosa bien distinta es que, a día de hoy, los docentes universitarios hayamos tomado conciencia real acerca de la aplicación de las dinámicas lúdicas, de juego, en el proceso de aprendizaje de nuestro estudiantado.

Concretamente, para que una actividad pueda ser considerada como "juego", y en consecuencia exista realmente una "gamificación" en la docencia, es indispensable que la actividad a "gamificar", ya se desarrolle de forma presencial o bien a través de la tecnología, cumpla siempre con un fin lúdico e incorpore, a su vez, una dinámica de “diversión”. Las dinámicas de juego persiguen implicar al estudiantado en el juego con el objetivo de que éste avance en la consecución de sus objetivos a medida que realiza la actividad "lúdica" (MUÑOZ BASOLS et al., 2019, 52). Puede hablarse, ciertamente, de la aplicación de una dinámica de recompensa con la que conseguir que los estudiantes universitarios, como ya se ha experimentado en la educación infantil y primaria, se interesen en la actividad que están realizando, bien sea reconociéndoles puntos positivos, reduciéndoles el número de preguntas de la prueba final de síntesis o eximiéndoles, parcialmente, de la realización de alguna otra actividad propia del curso); así como, en otros casos, también puede optarse por la implementación de una "dinámica de competición", dirigida a centralizar la atención en el interés y/o satisfacción por ganar o, en su caso, no quedar el último. A todas ellas suele también añadirse, en el ámbito educativo, una dinámica de "trabajo en equipo", pues no es nada infrecuente que en una actividad competitiva se puedan trabajar, en paralelo, las competencias de la cooperación y la solidaridad entre los miembros que integran el equipo de trabajo (KAPP, 2012, 34).

Llegados a este punto, con todo, resulta oportuno señalar algunos errores que, en el terreno de la "gamificación", es posible cometer con más frecuencia de la deseada. En 
este sentido, la tendencia por la "gamificación" puede conducir a la necesidad de adaptar o transformar todo aquello de lo que se dispone en formato físico al mundo digital. Así, por ejemplo, bien podría pasar que, aun manteniendo la misma estructura docente, sustentada en clases magistrales, se optase por diseñar la prueba de evaluación mediante la utilización de un cuestionario online, sustitutivo del tradicional formulario test en soporte papel, que los alumnos deben contestar desde su tablet o smartphone. Sin embargo, en estos casos, no puede hablarse con propiedad de una "gamificación" del aprendizaje, ya que no se cumplen los requisitos esenciales que toda actividad de "gamificación" exige, esto es, no se ha integrado contenido de conocimiento alguno en la actividad y tampoco se ha incorporado ninguna dinámica de juego (MUÑOZ BASOLS et al., 2019, 81-87). En este caso únicamente se ha apostado por la realización de una prueba de evaluación en formato digital.

\section{Experiencia personal de "gamificación" en el Grado de ADE de la Universitat de Barcelona (curso académico 2018/2019)}

Centrando la reflexión en el entorno de la ciencias económicas y jurídicas, cabe señalar que la "gamificación" siempre ha estado presente en las aulas universitarias, bien a través de juegos de mesa, de la construcción de un trivial de preguntas, de actividades divididas por estaciones, o de la elaboración de puzles con definiciones y conceptos. Con todo, la proliferación de grupos especialmente numerosos (de 70 a 100 alumnos) en este ámbito de conocimiento ha hecho incrementar el interés por organizar la actividad de "gamificación" mediante la aplicación de apps y otros recursos tecnológicos (MARTÍNEZ, 2017, 255-256).

Ante esta realidad y en lo que constituye la asignatura de Derecho Mercantil del Grado de ADE, a continuación se detallará la experiencia docente sobre el uso y dinámica de "gamificación" que, en todo caso, se ha empleado de modo ocasional y ha resultado útil para combatir la clásica desmotivación de parte del estudiantado, así como la difícultad y complejidad propia de una materia jurídica con notables implicaciones en el terreno de la economía y el mundo de la empresa.

En la estructuración de la docencia de esta asignatura se descartó, a diferencia de lo que acontece con el estudio del Derecho en los centros propios y especializados, la utilización del método del aprendizaje basado en problemas (ABP) o del aula inversa (FONT, 2011, 32-36). Ello ha respondido a diferentes factores críticos que merecen ser tenidos en consideración. De una parte, los estudiantes de primer curso no se han 
adaptado todavía a la dinámica universitaria. En esta etapa de transición académica, a la mayoría de ellos les resulta especialmente difícil acostumbrarse a trabajar, en un primer momento, de forma autónoma, máxime cuando se trata de una asignatura jurídica que se imparte en un grado, el de ADE, en el que predominan los conocimientos propios de las matemáticas, la economía y la estadística, motivo éste por el que el estudiantado no solo no está familiarizado con el mundo del Derecho, sino que, además, tampoco está habituado a trabajar con el soporte de los tradicionales manuales jurídicos (cabe decir que ante manuales de notable extensión, con mucha letra y casi nulas fotografías o fórmulas, sufren una cierta "desesperación"). De otra, la motivación que tienen los estudiantes es significativamente baja, pues parten del prejuicio, equivocado, de que todo lo jurídico es ajeno, por definición, al ámbito profesional de la empresa y los negocios (VÁZQUEZ, 2001, 11-12).

Es por ello, precisamente, que de forma consciente y voluntaria se ha optado, en cuanto estrategia metodológica de aprendizaje, por combinar las tradicionales "clases magistrales", orientadas a la fijación de los contenidos esenciales, con la incorporación de la "resolución de problemas" y la introducción en el aprendizaje de diferentes elementos que son propios de la "gamificación", con la intención de que la aplicación simultánea y continuada de todos ellos generase una dinámica que fuera capaz de invitar al alumno a mantener un proceso de aprendizaje activo y continuado en el tiempo.

Con tal de involucrar continuamente a los estudiantes en las sesiones de contenido más teórico se ha reemplazado la clase magistral tradicional por otra, aún de carácter teóricoexpositivo, en la que se parte en el comienzo de la sesión de un planteamiento inicial sobre un escenario de actualidad (ya sea un titular de noticia, un ejemplo hipotético planteado, o cualquier fragmento de película o anuncio) que permita trabajar los conceptos de cada bloque temático, pero, a su vez, pueda resultar cercano y familiar a la experiencia personal de los estudiantes. No obstante, los alumnos deben igualmente consolidar y completar dicha explicación con su trabajo autónomo.

A continuación, entre la primera sesión teórica del curso y la segunda, de naturaleza práctica y centrada en la resolución de un escenario-problema, se han introducido algunos elementos propios de la "gamificación" para que, además de reforzar los conocimientos adquiridos dentro del aula, empujen al alumno, de forma atractiva, a continuar con su proceso de aprendizaje fuera de ella. 
De esta forma, tras la finalización de cada sesión teórico-expositiva, los estudiantes disponen de un plazo breve de 24 horas para realizar a través del Campus Virtual dos tipos de actividades. En primer lugar, deben realizar un mapa conceptual sobre los contenidos de cada unidad didáctica analizada en la sesión presencial (indicando el fundamento o artículo legal, si lo hubiere). Este mapa conceptual se realiza a través de la aplicación $C$-MAP y debe subirse en la tarea abierta a tal fin en el Campus Virtual dentro del plazo habilitado. En segundo lugar, también deben realizar una segunda actividad, que consiste en realizar un cuestionario tipo test de 10 preguntas de respuesta múltiple.

Debe indicarse que tanto la implementación de los mapas y esquemas conceptuales como los test virtuales han coadyuvado a consolidar el proceso de aprendizaje. La elaboración de un mapa o esquema conceptual en cada unidad didáctica ha resultado de doble utilidad, pues ha servido, desde la perspectiva del docente, como evidencia de aprendizaje de que el alumno al menos ha tenido una toma de contacto inicial con los contenidos trabajados en clase en sus horas de trabajo autónomo, y desde la visión de los propios alumnos, han manifestado que les ha resultado útil para la realización de las prácticas (en la medida que disponen de un íter organizado y sistematizado a modo de guión o pauta para resolver y argumentar con mayor coherencia el caso práctico).

Por lo que respecta al empleo de los tests virtuales, también han tenido una buena respuesta por parte de los alumnos porque les ha servido, primero, como herramienta de gestión con la que organizar una distribución semanal de su carga de trabajo fuera del aula, facilitándoles el seguimiento regular del curso; y en segundo lugar, de haber cumplido honestamente con esa obligación, los cuestionarios online además les han resultado beneficiosos en la medida en que les han proporcionado un feedback inmediato sobre la adquisición correcta o no de los contenidos. A este último fin ha contribuido significativamente la configuración de las respuestas del test con la apertura de un cuadro de diálogo en la que se facilita un texto indicando la fundamentación y motivo de cada una de las respuestas.

Por otro lado, también se ha hecho uso de otros dos tipos de actividades "gamificadas", si bien de carácter más informal y no evaluables. De una parte, se han introducido juegos adaptados al contenido jurídico de la materia (crucigramas, sopas de letras, "pasapalabras" o columnas de relacionar concepto/acto con definición), resultando ser un entretenimiento y excusa perfecta para repasar las diferentes definiciones y 
categorías conceptuales con la finalización de cada bloque temático. De otra, se han introducido cuestionarios a través del kahoot con el objetivo de comprobar la correcta asimilación de los contenidos fundamentales de cada bloque temático.

Los kahoots, en particular, han tenido una muy buena acogida por el estudiantado. Además de ser una herramienta muy fácil de introducir en clase y promover la interacción social, ha resultado especialmente útil para complementar las clases de dudas y repaso de contenidos en las que los alumnos no se muestran altamente participativos ni proclives a expresar en público dudas o conceptos mal entendidos. Gracias al uso de kahoots se ha podido detectar como mayor facilidad, y dentro de un clima más distendido y lúdico, la existencia de errores conceptuales comúnmente compartidos por el grupo con el fin de corregirlos antes de iniciar el estudio final de la asignatura. Además, la propia disposición del programa resulta muy útil por cuanto permite parar el desarrollo del test y, en consecuencia, compaginar el juego con las aclaraciones o puntualizaciones que la profesora o profesor estime oportunas en cada caso concreto.

\section{Conclusiones}

Las metodologías docentes activas no son la panacea, pero tampoco algo a ignorar o desconocer. En consecuencia, el profesorado viene obligado, en aras de la calidad docente, a saber combinar lo mejor de la tradición con las exigencias que son propias de la modernidad, pero sin confundir ésta con la simple adaptación digital de lo que, desde siempre, habían sido actividades ya existentes en un formato más tradicional. En esta línea, resulta imprescindible que todo profesor o profesora de universidad, en el momento de "planificar su docencia", goce de libertad para combinar, bajo criterios racionales, las metodologías docentes clásicas (en especial, la clase magistral), de notable utilidad para transmitir conocimientos, con aquellas otras de naturaleza activa en las que el alumno está llamado a asumir un compromiso más participativo en su proceso de aprendizaje y que, sin duda, son útiles no solo para "consolidar" conocimientos, sino también para fomentar la adquisición de competencias y habilidades necesarias para el ejercicio de su profesión.

En esta dinámica, la "gamificación" puede y debe ser un complemento muy útil en orden a corregir algunas de las deficiencias a las que todo docente debe enfrentarse a diario en las aulas universitarias, como la desmotivación de parte del estudiantado o falta de asistencia y ausencia de implicación del estudiantado en su dinámica de 
aprendizaje. Ahora bien, debe aplicarse con sentido común y en el bien entendido que, por sí misma y de forma aislada, no debiera convertirse, bajo ningún concepto, en una estrategia educativa única y principal.

En este caso en particular, la combinación conjunta de las sesiones presenciales con la resolución de problemas y, la introducción de diferentes elementos de "gamificación" ha permitido mejorar algunos de los principales puntos críticos que tradicionalmente arrastraba la impartición de esta asignatura. De acuerdo con la recopilación de las diferentes fuentes de datos (listas de asistencias, número y continuidad de actividades entregadas y cuestionarios de incidencias críticas), así como también teniendo en consideración la percepción del docente y la retroalimentación facilitada por los propios estudiantes, se ha podido contrastar como la combinación de los elementos anteriores ha contribuido a una mejora de las calificaciones obtenidas por parte de los estudiantes, incrementándose tanto las calificaciones de las actividades de evaluación continuada (la nota media de todas las actividades del curso anterior se situaba en un 6.40 mientras que en el curso actual ha alcanzado el 7.44), como la calificación de la actividad final de síntesis (de un 4.58 del curso anterior hasta alcanzar un 5.55 en el curso docente actual), por lo que se advierte que el rendimiento académico no solo se ha incrementado sino que además ha sido sostenido durante el desarrollo de toda la asignatura.

Otro dato que pudiera resultar indicativo del impacto positivo que han tenido las medidas anteriores en su empuje por mantener un proceso de aprendizaje continuado guarda relación con el incremento del número de estudiantes que han decidido permanecer, en comparación con el del año anterior, en el régimen de evaluación continuada. En este sentido, ha disminuido significativamente el trasvase de alumnos hacia el régimen de evaluación única por motivos no laborales o personales, reduciéndose de las 14 solicitudes en el año académico anterior -sobre un total de 102 estudiantes- a una única petición en el curso actual - de un total de 80 estudiantes. De igual forma, también se ha apreciado un incremento notable de la asistencia a clase (pasando de un promedio de 27 estudiantes del curso anterior a uno de 46 en el actual).

En mi experiencia personal debo significar, como puede inferirse de los resultados, que la "gamificación" ha servido para que los alumnos se involucren en su propio proceso de aprendizaje; se vean abocados a llevar a cabo un estudio continuado y periódico a lo largo del curso (lo que es coherente con la generalización del sistema de evaluación continuada); y consigan familiarizarse, de forma atractiva y no aburrida, con los 
complejos contenidos teórico-prácticos que son propios de la asignatura de Derecho Mercantil impartida en el Grado de ADE a exhibir, de entrada, un formato de presentación más atractivo y cercano que el propio de la práctica tradicional.

\section{Referencias bibliográficas}

ANDRÉS AUCEJO, E. (2012). "La formación inicial de Jueces y Magistrados: nuevas metodologías de aprendizaje durante el período de formación en la Escuela Judicial", Revista de educación y derecho - Education and Law Review, 6, pp. 1-7.

BENITO MUNDET, H. et al. (2018). "Impacto de la innovación en la docencia y el aprendizaje", en S. Carrasco e I. del Corral (coords.), Docencia universitaria $e$ innovación: evolución y retos a través de los CIDUI. Barcelona: Octaedro, pp. 153-175.

BUXARRAIS ESTRADA, M. A. y BURGUET I ARFELIS, M. (2013). "La eticidad de las TIC: las competencias transversales y sus paradojas", Education in the knowledge society, $14(3)$, pp. 87-100.

CANTADOR, I. (2016). "La competición como mecánica de gamificación en el aula: una experiencia aplicando aprendizaje basado en problemas y aprendizaje cooperativo", en R. S. Contreras y J. L. Eguia (coords.), Gamificación en aulas universitarias. Barcelona: Institut de Comunicació UAB, pp. 67-96.

CÁRCEL CARRASCO, F. J. (2016). "Desarrollo de habilidades mediante el aprendizaje autónomo", 3c Empresa: investigación y pensamiento crítico, 5 (3), pp. 52 60.

CONTRERAS ESPINOSA, R. S. (2016). "Elementos de juego y motivación: reflexiones en torno a una experiencia que utiliza gamificación en una asignatura de grado para game designers", en R. S. Contreras y J. L. Eguia (coords.) Gamificación en las aulas universitarias. Barcelona: Institut de Comunicació UAB, pp. 55-66.

DOMINGO, J. (2008). El aprendizaje cooperativo, Cuadernos de trabajo social, 21, pp. 231-246.

ESCUDERO ESCORCA, T., RODRÍGUEZ, L. M. y FERNÁNDEZ, R. (2002). "Aprendizaje entre iguales y construcción de conceptos", Journal for the Study of Education and Development, 25 (3), pp. 279-285. 
ESTEVE, A. (2016). "Flipped Teaching o la clase invertida en la enseñanza del Derecho", Actualidad Jurídica Iberoamericana, 4 extra, pp. 75-95.

ESTEVE, F. y GISBERT, M. (2011). "El nuevo paradigma de aprendizaje y las nuevas tecnologías", Revista de Docencia Universitaria, 9 (3), pp. 55-69.

FONT RIBAS, A. (2014). "La enseñanza del derecho tras Bolonia", REDU: Revista de Docencia Universitaria, 12 (3 extra), pp. 36-41.

FONT RIBAS, A (2012). "El aprendizaje basado en problemas en la formación judicial”, Revista de Educación y Derecho - Education and Law Review, 6, pp. 1-20.

FONT RIBAS, A. (2011). "Diez años de experiencias con el grupo de innovación docente Dikasteia”, en M. Turull (coord.) Experiencias de mejora e innovación docente en el ámbito del Derecho. Barcelona: Octaedro ICE-UB, pp. 30-38.

GIFREU-CASTELLS, A. (2016). "Jugando en el aula con el documental interactivo y transmedia”, en R. S. Contreras y J. L. Eguia (coord.) Gamificación en las aulas universitarias. Barcelona: Institut de Comunicació UAB, pp. 96-110.

KAPP, K. (2012). The gamification of learning and instruction: Game-based methods and strategies for training and education. San Francisco: Pfeiffer.

MARTÍNEZ, M. (2006). "Formación para la ciudadanía y educación superior”, Revista iberoamericana de educación, 42, pp. 85-102.

MARTÍNEZ NAVARRO, G. (2017). “Tecnologías y nuevas tendencias en educación: Aprender jugando. El caso de Kahoot”, Opción: Revista de Ciencias Humanas y Sociales, 83, pp. 252-277.

MUÑOZ BASOLS, J. et al. (2019). The Routledge Handbook of Spanish Language Teaching: metodologías, contextos y recursos para la enseñanza del español L2. Lodon: Routledge.

NAVAL, C. et al. (2011). "Ethical and civic education and the social commitment of university students", Encounters on Education, 12, pp. 77-91.

PÉREZ CABRERA, L. (2013). "El rol del docente en el aprendizaje autónomo: la perspectiva del estudiante y la relación con su rendimiento académico", Revista Diálogos, 11, pp. 45-62.

PRENSKY, M. (2000). Digital Game-Based Learning. Minnesota: McGraw Hill. 
VALLESPÍN PÉREZ, D. (2013). "La gobernanza de la Universidad: el camino hacia un "cambio razonable" compatible con el respeto de la autonomía universitaria y la libertad de cátedra", Revista Educación y Derecho - Education and Law Review, 5, pp. 8-15.

VALLESPÍN PÉREZ, D. (2011). "El método del caso como instrumento de aprendizaje en el Derecho Procesal", Revista Educación y Derecho - Education and Law Review, 3, pp. 1-10.

VÁZQUEZ ALBERT, D. et. al. (2011). “La enseñanza reflexiva del Derecho Mercantil: el diálogo reflexivo y la interrogación didáctica", en M. Turull (coord.) Experiencias de mejora e innovación docente en el ámbito del Derecho. Barcelona: Octaedro ICE -UB, pp. 49-59. 